\title{
Detecting abnormal methylation of tumor suppressor genes GSTP1, P16, RIZ1, and RASSF1A in hepatocellular carcinoma and its clinical significance
}

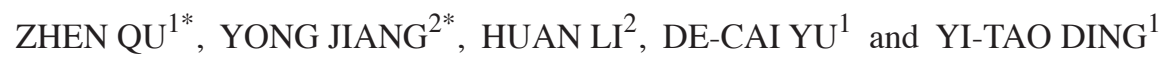 \\ ${ }^{1}$ Department of Hepatobiliary Surgery, Drum Tower Hospital, Medicine School of Nanjing University, \\ Nanjing, Jiangsu 210008; ${ }^{2}$ Department of Hepatobiliary Surgery, Changzhou First People's Hospital, \\ The Third Affiliated Hospital of Soochow University, Changzhou, Jiangsu 213003, P.R. China
}

Received October 21, 2014; Accepted July 7, 2015

DOI: $10.3892 / 01.2015 .3536$

\begin{abstract}
Hepatocellular carcinoma (HCC) has a high rate of mortality. Further studies into epigenetic changes in HCC, particularly the abnormal methylation of tumor suppressor genes (TSGs), are required, since these changes may provide novel biomarkers for early screening and diagnosis of HCC. By using methylation-specific polymerase chain reaction (MSP), the present study detected the methylation status in the promoter region of 4 candidate TSGs, GSTP1, P16, RIZ1, and $R A S S F 1 A$, respectively, in 35 paired HCC and tumor-adjacent liver tissues in addition to 20 normal liver tissues. Their effect on the initiation and progression of HCC was also investigated by analyzing the clinicopathological data. The results of the present study revealed that the methylation level of RIZl and GSTPl genes in HCC was significantly increased compared with that in the adjacent tissues $(\mathrm{P}<0.01)$ and the normal liver tissues $(\mathrm{P}<0.01)$. The methylation frequency of $P 16$ and RASSF1A genes was not significantly increased compared with that observed in the adjacent tissues $(\mathrm{P}>0.05)$ but was significantly increased compared with the normal tissues $(\mathrm{P}<0.01)$. In HCC tissues, the methylation frequency of the GSTPI gene in tumors with capsular invasion was significantly increased compared with that in tumors without capsular invasion $(\mathrm{P}<0.05)$. The methylation frequency of $\mathrm{P} 16$ gene in hepatitis B surface antigen ( $\mathrm{HbsAg}$ )-positive HCC patients was significantly increased compared with that in HbsAg-negative patients $(\mathrm{P}<0.05)$. The methylation status of RIZ1 and RASSF1A genes was not significantly correlated with
\end{abstract}

Correspondence to: Professor Yi-Tao Ding, Department of Hepatobiliary Surgery, Drum Tower Hospital, Medicine School of Nanjing University, 321 Zhongshan Road, Nanjing, Jiangsu 210008, P.R. China

E-mail: drdingyitao0@sina.com

*Contributed equally

Key words: hepatocellular carcinoma, suppressor gene, methylation the clinicopathological data $(\mathrm{P}>0.05)$. Previous studies have demonstrated that the methylation status of RIZ1 and GSTP1 genes is HCC-specific, and thus may be used as a biomarker to assist the clinical diagnosis of HCC. While the methylation of GSTP1 gene promoter may associate with the invasiveness of $\mathrm{HCC}$, chronic hepatitis B virus infection may be the cause of methylation-induced $P 16$ inactivation.

\section{Introduction}

HCC is one of the tumors with the highest incidence worldwide, and its incidence and mortality in China have remained high. The occurrence of HCC is results from multiple factors, including the activation of certain oncogenes, inactivation of TSGs and exogenous stimuli. Previous studies have demonstrated that the hypermethylation of $\mathrm{CpG}$ islands in tumor suppressor gene (TSG) promoters is closely associated with the formation of HCC, which may transform the spatial structure of chromatin and hence block/silence the transcription of TSGs via recruiting proteins of the methyl $\mathrm{CpG}$ binding domain (MBD) family in addition to the associated protein complexes $(1,2)$. Epigenetic silencing is a relatively common mechanism of TSG inactivation (3). The aberrant methylation of tumor-associated genes, particularly TSGs, requires further research.

A high frequency of methylation inactivation of the GSTPI gene has been observed in human prostate, kidney, and liver cancers (4-6). Zhang et al (7) and Tchou et al (8) demonstrated that there is a high frequency of methylation events in the GSTP1 gene in HCC tumor samples and HCC cell lines, and that the methylation of GSTPI in HCC is associated with the action of environmental carcinogens. As a TSG, P16 gene inactivation may result in excessive cell proliferation, and the promoter methylation on 9p21 in HCC patients represents the most common mechanism of P16 inactivation (9). Zhong et al (10) revealed that the abnormal methylation of the RASSF1A gene promoter is present in $95 \%$ of HCC tissues; the authors hypothesized that the change in RASSF1A gene expression is an early event during hepatitis B virus-induced tumorigenesis of HCC.

The present study comparatively analyzed the changes in methylation level of 4 TSGs in samples from HCC tumors, tumor-adjacent tissues, and normal liver tissues, and 
investigated their correlation with the occurrence and progression of HCC by consulting the clinicopathological data, in order to provide a novel way for the early screening and gene diagnosis and therapy of liver cancer.

\section{Materials and methods}

Written informed consent was obtained from the families of all patients, and the Human Research Ethics Committee of the Affiliated Nanjing University Drum Tower Hospital (Nanjing, China) approved the use of all samples under a protocol that conforms to the provisions of the Declaration of Helsinki (as revised in Seoul, 2008).

Specimens. The tumor specimens were collected from HCC patients who had undergone surgical treatment in the Department of Hepatobiliary Surgery of the Affiliated Drum Tower Hospital, School of Medicine, Nanjing University, in the Changzhou First People's Hospital, and in the Third Affiliated Hospital of Soochow University, during the period between January 2013 and January 2014. The patients did not receive any anticancer treatment and had no other endocrine, immune, and metabolic diseases prior to the surgery. Any hemorrhagic and necrotic regions were avoided during the tumor sample collection. The tumor-adjacent liver tissues were obtained from the area within $1.5 \mathrm{~cm}$ distance from the edge of $\mathrm{HCC}$, and histologically confirmed for no infiltration of cancer cells. The normal control group contained 20 cases of pathologically confirmed normal liver tissue. All the specimens were frozen in liquid $\mathrm{N}_{2}$ immediately following the resection, then transported, and stored at $-80^{\circ} \mathrm{C}$.

Methylation-specific polymerase chain reaction (MSP) and result interpretation. The DNA samples were extracted from the liver specimens using a DNA extraction kit (Sangon Biotech Shanghai Co., Ltd., Shanghai, China), following the manufacturer's instructions. The concentration and purity of the extracted DNA were measured on a UV spectrophotometer (UV-240; Shimadzu Corp., Kyoto, Japan), and suitable DNA samples were stored at $-80^{\circ} \mathrm{C}$.

Bisulfite modification of the DNA samples was conducted using anEZ DNA Methylation-Direct ${ }^{\mathrm{TM}}$ Kit (Zymo Research, Irvine, CA, USA) according to the kit instructions. The DNA samples were then amplified by MSP and analyzed for methylation status based on the differential amplification pattern.

The primer sequences, annealing temperature, and product sizes are presented in Table I. The PCR system contained PCR Mixture 2X Mix $15 \mu 1, \mathrm{U}$ or M-Primer F $0.5 \mu \mathrm{l}, \mathrm{U}$ or M-Primer R $0.5 \mu$, Modified DNA 1-5 $\mu \mathrm{l}$; the total volume was adjusted to $30 \mu \mathrm{l}$ with deionized $\mathrm{H}_{2} \mathrm{O}$. The PCR conditions were as follows: $94^{\circ} \mathrm{C}$ denaturation, $3 \mathrm{~min} ; 94^{\circ} \mathrm{C}$ denaturation $30 \mathrm{sec}$, annealing $30 \mathrm{sec}$, and $72^{\circ} \mathrm{C}$ extension $30 \mathrm{sec}, 40$ cycles; $72^{\circ} \mathrm{C}$ extension $7 \mathrm{~min}$. The amplification product was stored at $4^{\circ} \mathrm{C}$. The PCR products (10 $\mu \mathrm{l}$ each) were separated by $2 \%$ agarose gel electrophoresis, and the images were captured on a gel imaging and analysis system (Yu Long Co., Ltd., Kunming, China).

Samples that amplified from the primer pair for methylated DNA sequence were considered methylation-positive, whereas samples that amplified from the primer pair for unmethylated sequence were considered methylation-negative. Samples with

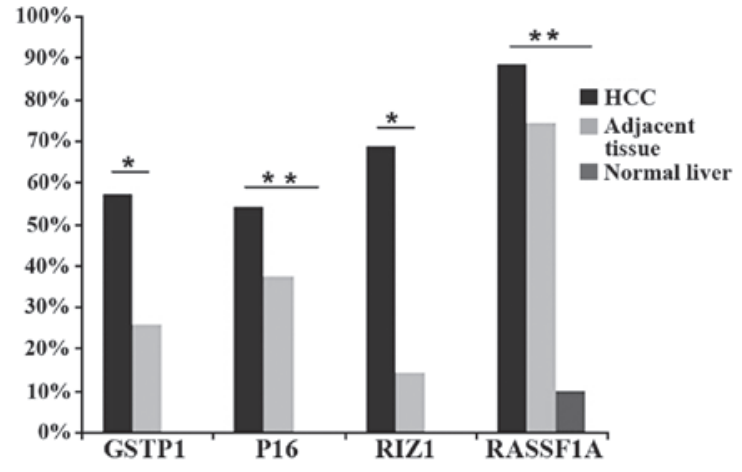

Figure 1. The status of methylation of GSTP1, P16, RIZ1, and RASSF1A genes in the 3 groups of tissues. ${ }^{*} \mathrm{P}<0.05$. ${ }^{* *} \mathrm{P}<0.01$.

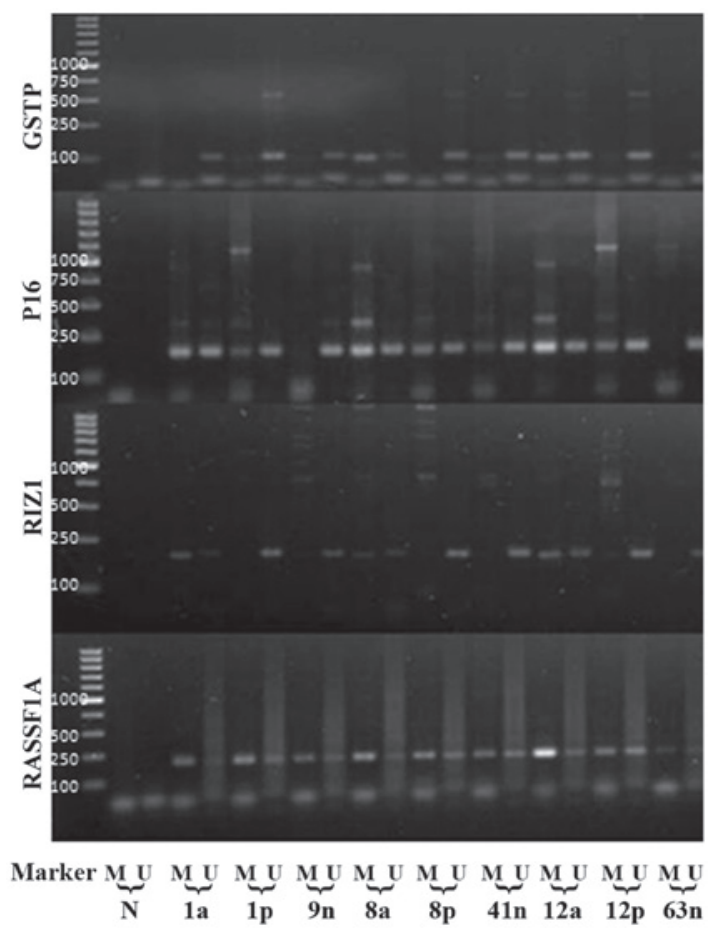

Figure 2. The MSP products of GSTP1, P16, RIZ1, and RASSF1A genes in part of the HCC, paired tumor-adjacent, and normal liver tissues. The lowest marker band represents $100 \mathrm{bp}$. N, PCR negative control; M, methylated; and $\mathrm{U}$, unmethylated. a, cancerous tissue; $\mathrm{p}$, adjacent liver tissue; and n, normal liver tissue.

PCR products from both primer pairs were considered partially methylated, which also represents a methylation-positive status.

Statistical analysis. The data were statistically analyzed using SPSS statistical software, version 17.0 (SPSS, Inc., Chicago, IL, USA). The count and measurement data were compared by $\chi^{2}$ test and $t$-test respectively. The association between the gene methylation in HCC tissues and the clinical data was analyzed using a Fisher's exact probability test. $\mathrm{P}<0.05$ was used to indicate a statistically significant difference.

\section{Results}

The frequency of methylation of GSTP1 and RIZ1 genes in HCC tissues were $57.1 \%$ (20/35) and 68.6\% (24/35) respectively, 
Table I. Primers for methylation-specific polymerase chain reaction.

\begin{tabular}{|c|c|c|c|c|}
\hline Gene & $\mathrm{M} / \mathrm{U}$ & Primer sequence $\left(5^{\prime}-3^{\prime}\right)$ & Annealing temperature & Product length \\
\hline \multirow[t]{2}{*}{ GSTP1 } & M & $\begin{array}{l}\text { (F)-TTCGGGGTGTAGCGGTCGTC } \\
\text { (R)-GCCCCAATACTAAATCACGACG }\end{array}$ & 61 & 91 \\
\hline & $\mathrm{U}$ & $\begin{array}{l}\text { (F)-GATGTTTGGGGTGTAGTGGTTGTT } \\
\text { (R)-CCACCCCAATACTAAATCACAACA }\end{array}$ & 55 & 91 \\
\hline \multirow[t]{2}{*}{ P16 } & M & $\begin{array}{l}\text { (F)-TTATTAGAGGGTGGGGCGGATCGC } \\
\text { (R)-CAACCCCAAACCACAACCATAA }\end{array}$ & 60 & 150 \\
\hline & $\mathrm{U}$ & $\begin{array}{l}\text { (F)-TTATTAGAGGGTGGGGTGGATTGT } \\
\text { (R)-CAACCCCAAACCACAACCATAA }\end{array}$ & 55 & 151 \\
\hline \multirow[t]{2}{*}{$R I Z 1$} & M & $\begin{array}{l}\text { (F)-GGATTCGCGGTGATTTAC } \\
\text { (R)-AACTCCAATCGAAAAAAACG }\end{array}$ & 69 & 161 \\
\hline & $\mathrm{U}$ & $\begin{array}{l}\text { (F)-ATGGGATTTGTGGTGATTTAT } \\
\text { (R)-CTTAACTCCAATCAAAAAAAACA }\end{array}$ & 58 & 161 \\
\hline \multirow[t]{2}{*}{ RASSF IA } & M & $\begin{array}{l}\text { (F)-GGGTTTTGCGAGAGCGC } \\
\text { (R)-CGCTAACAAACGCGAACCG }\end{array}$ & 52 & 169 \\
\hline & $\mathrm{U}$ & $\begin{array}{l}\text { (F)-GGTTTTGTGAGAGTGTGTTTAG } \\
\text { (R)-CACTAACAAACACAAACCAAAC }\end{array}$ & 52 & 169 \\
\hline
\end{tabular}

M, methylated, U, unmethlyated.

Table II. The methylation status of GSTP1, P16, RIZ1, and RASSF1A genes.

\begin{tabular}{|c|c|c|c|c|c|c|c|c|}
\hline \multirow[b]{3}{*}{ Group } & \multicolumn{8}{|c|}{ Methylation status } \\
\hline & \multicolumn{2}{|c|}{ GSTP1 } & \multicolumn{2}{|c|}{ P16 } & \multicolumn{2}{|c|}{$R I Z 1$} & \multicolumn{2}{|c|}{ RASSF $1 A$} \\
\hline & + & - & + & - & + & - & + & - \\
\hline $\operatorname{HCC}(n=35)$ & 20 & 15 & 19 & 16 & 24 & 11 & 31 & 4 \\
\hline Adjacent tissue $(n=35)$ & 9 & 26 & 13 & 22 & 5 & 30 & 26 & 9 \\
\hline Normal liver $(n=20)$ & 0 & 20 & 0 & 20 & 0 & 20 & 2 & 18 \\
\hline $\mathrm{P}$-value & $0.008^{\mathrm{a}}$ & $0.036^{\mathrm{b}}$ & $0.150^{\mathrm{a}}$ & $0.005^{\mathrm{b}}$ & $<0.01^{\mathrm{a}}$ & $0.199^{\mathrm{b}}$ & $0.124^{\mathrm{a}}$ & $<0.01^{\mathrm{b}}$ \\
\hline
\end{tabular}

${ }^{\mathrm{a}}$ Comparison between HCC and tumor-adjacent tissues; ${ }^{\mathrm{b}}$ comparison between HCC and normal liver tissues.

both of which were significantly increased $(\mathrm{P}<0.01)$ compared with the levels in adjacent liver tissues $(25.7 \%$ [9/35] and $14.3 \%$ [5/35] respectively). The frequencies of methylation of $P 16$ and RASSF1A genes in HCC tissues were 54.3\% (19/35) and $88.6 \%$ (31/35), respectively, which did not significantly differ $(\mathrm{P}>0.05)$ from those in adjacent liver tissues (37.1\% [13/35] and $74.3 \%$ [26/35]). No methylation of the GSTP1, P16, and $R I Z 1$ genes was observed in the 20 cases of normal liver tissue, and the methylation of RASSF1A gene was identified in $10 \%$ (2/20) normal liver tissues (Table II, Figs. 1 and 2).

The frequency of GSTP1 gene methylation in HCC with capsular invasion was significantly increased compared with in $\mathrm{HCC}$ without capsular invasion $(\mathrm{P}<0.05)$. Among the HCC samples, the frequency of P16 gene methylation in $\mathrm{Hbs} \mathrm{Ag}$-positive HCC tissues was significantly increased compared with those in HbsAg-negative HCC tissues $(\mathrm{P}<0.05)$. The methylation status of $R I Z 1$ and
RASSF1A genes did not demonstrate significant correlation with the clinicopathological data of patients $(\mathrm{P}>0.05$; Tables III and IV).

\section{Discussion}

HCC is one of the tumors with highest incidence worldwide. Due to an insidious onset, the majority of HCC patients are diagnosed at an advanced stage, which results in a $<20 \%$ clinically resectable rate $(11,12)$. With the exception of $\alpha$-fetoprotein (AFP), at present, there remains a lack of well-recognized effective tumor markers for routine clinical detection of HCC. Therefore, identifying HCC-associated tumor markers and studying the underlying molecular mechanisms is of great importance. Epigenetics refers to a genetic mechanism that enables the occurrence of inheritable alterations in gene expression without changing the DNA sequence (3). Epigenetic 
Table III. The correlation between the methylation state of GSTP1 and P16 genes with the clinicopathological data of HCC patients.

\begin{tabular}{|c|c|c|c|c|c|c|}
\hline \multirow[b]{2}{*}{ Clinicopathology } & \multicolumn{3}{|c|}{ GSTP1 methylation } & \multicolumn{3}{|c|}{ P16 methylation } \\
\hline & - & + & P-value & - & + & P-value \\
\hline Gender & & & 0.473 & & & 0.452 \\
\hline Male & 11 & 16 & & 13 & 14 & \\
\hline Female & 4 & 4 & & 3 & 5 & \\
\hline Age (years) & & & 0.281 & & & 0.364 \\
\hline$\leq 50$ & 6 & 5 & & 6 & 5 & \\
\hline$>50$ & 9 & 15 & & 10 & 14 & \\
\hline $\operatorname{AFP}(\mu \mathrm{g} / \mathrm{l})$ & & & 0.518 & & & 0.156 \\
\hline$\leq 20$ & 6 & 7 & & 4 & 9 & \\
\hline$>20$ & 9 & 13 & & 12 & 10 & \\
\hline Tumor size $(\mathrm{cm})$ & & & 0.404 & & & 0.210 \\
\hline$\leq 5$ & 9 & 10 & & 7 & 12 & \\
\hline$>5$ & 6 & 10 & & 9 & 7 & \\
\hline Cirrhosis & & & 0.482 & & & 0.347 \\
\hline+ & 10 & 12 & & 9 & 13 & \\
\hline - & 5 & 8 & & 7 & 6 & \\
\hline HbsAg & & & 0.668 & & & 0.024 \\
\hline+ & 12 & 16 & & 10 & 18 & \\
\hline - & 3 & 4 & & 6 & 1 & \\
\hline Capsular invasion & & & 0.017 & & & 0.596 \\
\hline+ & 5 & 15 & & 9 & 11 & \\
\hline - & 10 & 5 & & 7 & 8 & \\
\hline Distal metastasis & & & 0.567 & & & 0.481 \\
\hline+ & 4 & 6 & & 4 & 6 & \\
\hline - & 11 & 14 & & 12 & 13 & \\
\hline Differentiation & & & 0.340 & & & 0.602 \\
\hline High to medium & 13 & 15 & & 13 & 15 & \\
\hline Poor & 2 & 5 & & 3 & 4 & \\
\hline
\end{tabular}

HCC, hepatocellular carcinoma; AFP, $\alpha$-fetoprotein; HbsAg, hepatitis B surface antigen.

silencing may result in the inactivation of TSGs, thereby causing the initiation and progression of tumorigenesis (3). The aberrant methylation of tumorigenesis-associated genes, particularly the TSGs, are receiving more attention.

GSTP1 gene is located on human chromosome 11q13, encoding an enzyme with detoxification and protein-binding functions (13). Previous studies have demonstrated that the high-frequency of methylation inactivation of GSTP1 gene is restricted to certain human cancers, including prostate, kidney, breast, and liver cancers $(4,5)$. Another previous study demonstrated that the frequency of GSTPl gene methylation in HCC is $41-85 \%$, and that the methylation of GSTPl gene in HCC is associated with the effect of environmental carcinogens (7). The frequency of GSTP1 gene methylation in HCC and its cell lines may be as high as $\sim 85 \%$, which is significantly increased compared with those in abnormal proliferation-induced liver nodules and cirrhosis tissues (8). The present study demonstrated that the frequency of GSTP1 methylation in
HCC $(57.1 \%$ [20/35]) was significantly increased $(\mathrm{P}<0.01)$ compared with that in tumor-adjacent tissues $(25.7 \%$ [9/35]), while no abnormal methylation was detected in normal liver tissues. The association between GSTP1 methylation in HCC and the clinicopathological data was investigated, and the results demonstrated that the frequency of GSTP1 methylation in HCC with capsular invasion was significantly increased compared with that in HCC without capsular invasion $(\mathrm{P}<0.05)$, while no significant correlation between other clinicopathological features and GSTP1 methylation was detected $(\mathrm{P}>0.05)$. Capsular invasion is associated with the metastasis and invasion of tumor. GSTP1 gene promoter methylation interferes with its normal expression or function, leading to an accumulation of $\beta$-catenin in the cells (14). Since the latter is important in mediating the epithelial cell adhesion, such change may therefore facilitate the intrahepatic metastasis of HCC (6). Therefore, the aberrant methylation of GSTPl gene may be associated with the invasiveness of HCC. 
Table IV. The correlation of the methylation state of RIZ1 and RASSF1A genes with the clinicopathological data of HCC patients.

\begin{tabular}{|c|c|c|c|c|c|c|}
\hline \multirow[b]{2}{*}{ Clinicopathology } & \multicolumn{3}{|c|}{ RIZ1 methylation } & \multicolumn{3}{|c|}{ RASSF $1 A$ methylation } \\
\hline & - & + & P-value & - & + & P-value \\
\hline Gender & & & 0.492 & & & 0.665 \\
\hline Male & 8 & 19 & & 3 & 24 & \\
\hline Female & 3 & 5 & & 1 & 7 & \\
\hline Age (years) & & & 0.521 & & & 0.372 \\
\hline$\leq 50$ & 3 & 8 & & 2 & 9 & \\
\hline$>50$ & 8 & 16 & & 2 & 22 & \\
\hline $\operatorname{AFP}(\mu \mathrm{g} / \mathrm{l})$ & & & 0.626 & & & 0.522 \\
\hline$\leq 20$ & 4 & 9 & & 1 & 12 & \\
\hline$>20$ & 7 & 15 & & 3 & 19 & \\
\hline Tumor size (cm) & & & 0.352 & & & 0.630 \\
\hline$\leq 5$ & 7 & 12 & & 2 & 17 & \\
\hline$>5$ & 4 & 12 & & 2 & 14 & \\
\hline Cirrhosis & & & 0.144 & & & 0.478 \\
\hline+ & 5 & 17 & & 2 & 20 & \\
\hline- & 6 & 7 & & 2 & 11 & \\
\hline $\mathrm{HbsAg}$ & & & 0.619 & & & 0.609 \\
\hline+ & 9 & 19 & & 3 & 25 & \\
\hline- & 2 & 5 & & 1 & 6 & \\
\hline Capsular invasion & & & 0.281 & & & 0.200 \\
\hline+ & 5 & 15 & & 1 & 19 & \\
\hline- & 6 & 9 & & 3 & 12 & \\
\hline Distal metastasis & & & 0.309 & & & 0.681 \\
\hline+ & 2 & 8 & & 1 & 9 & \\
\hline- & 9 & 16 & & 3 & 22 & \\
\hline Differentiation & & & 0.619 & & & 0.609 \\
\hline High to medium & 9 & 19 & & 3 & 25 & \\
\hline Poor & 2 & 5 & & 1 & 6 & \\
\hline
\end{tabular}

HCC, hepatocellular carcinoma; AFP, $\alpha$-fetoprotein; HbsAg, hepatitis B surface antigen.

As a TSG, the inactivation of P16 may lead to excessive cell proliferation, accelerated cell cycle, and hence a premature entry into the $\mathrm{S}$ phase prior to the completion of DNA repair, resulting in tumorigenesis. Jin et al (9) demonstrated that the promoter methylation on $9 \mathrm{p} 21$ is the most common mechanism for the inactivation of TSG P16 in HCC. Narimatsu et al (14) studied the methylation status of P16 in 35 cases of HCC infected by HBV and/or HCV using the MSP method; their results indicated that $P 16$ methylation may be induced by hepatitis virus in livers with chronic inflammation prior to the tumorigenesis of HCC. The present study demonstrated that the frequency of Pl6 gene methylation in HCC (54.3\% [19/35]) was increased compared with in the adjacent liver tissues $(37.1 \%$ [13/35]), though the difference was not statistically significant ( $\mathrm{P}>0.05$ ); there was no abnormal methylation of P16 detected in the normal liver tissues, implying a potential tumorigenic tendency of the tumor-adjacent tissues and a possible involvement of P16 methylation in the occurrence of HCC. Further statistical analysis of the association between the methylation results and the clinicopathological data of $\mathrm{HCC}$ indicated that the frequency of P16 methylation in HbsAg-positive patients was significantly increased compared with in HbsAg-negative ones $(\mathrm{P}<0.05)$, indicating that chronic HBV infection may be the result of methylation inactivation of $P 16$.

$R I Z 1$ is a TSG that simultaneously regulates cell proliferation and inhibits oncogenesis (15). The abnormal expression of RIZ1 is associated with the tumorigenesis of a variety of human tumors, including nasopharyngeal, breast, liver and cervical among other cancers $(16,17)$. Nomoto et al (18) reported that the frequency of $R I Z 1$ gene methylation was $45.2 \%$ among patients with HCC in Japan. The results of the present study demonstrated that the frequency of RIZ1 methylation in HCC $(68.6 \%$ [24/35]) was significantly increased $(\mathrm{P}<0.01)$ compared with in the paired adjacent liver tissues (14.3\% [5/35]), while no methylation of $R I Z 1$ was detected in the normal liver tissues. The frequency of RIZI methylation demonstrated a significant gradient among the 3 groups of samples; the rare presence of RIZI in the adjacent 
liver tissues indicated highly tumor-specific epigenetic changes in $R I Z 1$ with the occurrence of HCC, indicating that the methylation of $R I Z 1$ may be a frequent event during the tumorigenesis of HCC, and that the appearance of RIZ1 methylation may indicate an immediate formation of HCC. Therefore, RIZ1 gene methylation may be of great significance for the risk assessment and early diagnosis of HCC. However, further analysis indicated that there was no correlation between RIZ1 methylation and the clinicopathological data of HCC patients. It should be noted that since the exact role of $R I Z 1$ promoter methylation in the process HCC carcinogenesis remains unclear, further study with an expanded sample size is required.

RASSF1A is a TSG cloned from lung cancer and reported by Damman et al in 2000 (19). In certain cases RASSF1A is inactivated via abnormal promoter methylation in primary HCC. Zhong et al (10) demonstrated that the abnormal hypermethylation of the RASSF1A promoter sequence exists in $95 \%$ of HCC tissues, and proposed that RASSF1A methylation is a relatively early event during the $\mathrm{HBV}$-induced tumorigenesis of HCC. Previous studies have demonstrated that the frequency of RASSF1A gene methylation in HCC is $66.7-100 \%$, and the variation may be associated with ethnic and geographical variabilities $(20,21)$. In the present study, 31/35 $(88.6 \%)$ of HCC specimens presented with aberrant methylation, as did 26/35 (74.3\%) of the corresponding tumor-adjacent tissues; no statistically significant difference in aberrant methylation was observed between the above 2 groups ( $P>0.05)$. Taking into account that cancer is a systemic disease, the genetic and epigenetic anomalies occurring in the early stage of carcinogenesis may have already existed in the tumor-adjacent tissues. These results indicated that RASSFIA gene methylation may be involved during the occurrence and development of HCC, as an early event and most likely representing an early change in HCC. But given that a significant difference was not observed in the frequency of RASSF1A methylation in HCC and adjacent liver tissues, RASSFIA was not considered as one of the candidate auxiliary biomarkers for HCC diagnosis.

In summary, by analysis of the methylation frequency of 4 TSGs in HCC, tumor-adjacent, and normal liver tissues, the present study revealed a progressive epigenetic change during the formation of HCC, which reflected the molecular mechanism of the multi-step and multi-stage origin of HCC. In addition, the results demonstrated that the status of RIZI and GSTP1 gene methylation has good specificity for HCC, may better distinguish between HCC and non-cancerous tissues, and may therefore be used as novel candidate biomarkers to assist the early screening and diagnosis of HCC. With further study on TSG methylation and larger sample sizes in the future, a more thorough insight may be gained into the effect of abnormal methylation of TSGs on the occurrence and development of HCC.

\section{Acknowledgements}

The authors wish to acknowledge the excellent technical support of Dr Guang-Hua Luo and Dr Bao-Qiang Wu.

\section{References}

1. Bonasio R, Tu S and Reinberg D: Molecular signals of epigenetic states. Science 330: 612-616, 2010.
2. Herceg Z and Paliwal A: Epigenetic mechanisms in hepatocellular carcinoma: How environmental factors influence the epigenome. Mutat Res 727: 55-61, 2011.

3. Holliday R and Grigg GW: DNA methylation and mutation. Mutat Res 285: 61-67, 1993.

4. Yamanaka M, Watanabe M, Yamada Y, Takagi A, Murata T, Takahashi H, Suzuki H, Ito H, Tsukino H, Katoh T, et al: Altered methylation of multiple genes in carcinogenesis of the prostate. Int J Cancer 106: 382-387, 2003.

5. Hoque MO, Begum S, Topaloglu O, Jeronimo C, Mambo E, Westra WH, Califano JA and Sidransky D: Quantitative detection of promoter hypermethylation of multiple genes in the tumor, urine and serum DNA of patients with renal cancer. Cancer Res 64: 5511-5517, 2004.

6. Guan CN, Chen XM, Lou HQ, Liao XH, Chen BY and Zhang PW: Clinical significance of axin and $\beta$-catenin protein expression in primary hepatocellular carcinomas. Asina Pac J Cancer Prev 13: 677-681, 2012.

7. Zhang YJ, Chen Y, Ahsan H, Lunn RM, Chen SY, Lee PH, Chen CJ and Santella RM: Silencing of glutathione S-transferase P1 by promoter hypermethylation and its relationship to environmental chemical carcinogens in hepatocellular carcinoma. Cancer Lett 221: 135-143, 2005.

8. Tchou JC, Lin X, Freije D, Isaacs WB, Brooks JD, Rashid A, De Marzo AM, Kanai Y, Hirohashi S and Nelson WG: GSTP1 CpG island DNA hypermethylation in hepatocellular carcinomas. Int J Oncol 16: 663-676, 2000.

9. Jin M, Piao Z, Kim NG, Park C, Shin EC, Park JH, Jung HJ, Kim CG and Kim H: p16 is a major inactivation target in hepatocellular carcinoma. Cancer 89: 60-68, 2000.

10. Zhong S, Yeo W, Tang MW, Wong N, Lai PB and Johnson PJ: Intensive hypermethylation of the $\mathrm{CpG}$ island of Ras association domain family $1 \mathrm{~A}$ in hepatitis B virus-associated hepatocellular carcinomas. Clin Cancer Res 9: 3376-3382, 2003.

11. Yamane B and Weber S: Liver-directed treatment modalities for primary and secondary hepatic tumors. Surg Clin North Am 89: 97-113, 2009.

12. Delis SG, Bakoyiannis A, Tassopoulos N, Athanassiou K, Kelekis D, Madariaga $J$ and Dervenis C: Hepatic resection for hepatocellular carcinoma exceeding Milan criteria. Surg Oncol 19: 200-207, 2010 .

13. Nakazato H, Suzuki K, Matsui H, et al: Association of genetic polymorphisms of glutathione-S-transferase genes (GSTM1, GSTT1 and GSTP1) with familial prostate cancer risk in a Japanese population. Anticancer Res 23: 2897-2902, 2003.

14. Narimatsu T, Tamori A, Koh N, Kubo S, Hirohashi K, Yano Y, Arakawa T, Otani S and Nishiguchi S: p16 promoter hypermethylation in human hepatocellular carcinoma with or without hepatitis virus infection. Intervirology 47: 26-31, 2004.

15. Chadwick RB, Jiang GL, Bennington GA, Yuan B, Johnson CK, Stevens MW, Niemann TH, Peltomaki P, Huang S and de la Chapelle A: Candidate tumor suppressor RIZ is frequently involved in colorectal carcinogenesis. Proc Natl Acad Sci USA 97: 2662-2667, 2000.

16. Chen LB, Xu JY, Yang Z and Wang GB: Silencing SMYD3 in hepatoma demethylates RIZ1 promoter induces apoptosis and inhibits cell proliferation and migration. World $\mathrm{J}$ Gastroenterol 13: 5718-5724, 2007.

17. Chang HW, Chan A, Kwong DL, Wei WI, Sham JS and Yuen AP: Detection of hypermethylated RIZ1 gene in primary tumor, mouth and throat rinsing fluid, nasopharyngeal swab and peripheral blood of nasopharyngeal carcinoma patient. Clin Cancer Res 9: 1033-1038, 2003.

18. Nomoto S, Kinoshita T, Kato K, Otani S, Kasuya H, Takeda S, Kanazumi N, Sugimoto H and Nakao A: Hypermethylation of multiple genes as clonal markers in multicentric hepatocellular carcinoma. Br J Cancer 97: 1260-1265, 2007.

19. Damman R, Li C, Yoon JH, Chin PL, Bates S and Pfeifer GP: Epigenetic inactivation of RAS association domain family protein from the lung tumor suppressor locus 3p21.3. Nat Genet 25: 315-319, 2000.

20. Yu J, Ni M, Xu J, Zhang H, Gao B, Gu J, Chen J, Zhang L, $\mathrm{Wu} \mathrm{M}$, Zhen S and Zhu J: Methylation profiling of twenty promoter- $\mathrm{CpG}$ islands of genes which may contribute to hepatocellular carcinogenesis. BMC Cancer 2: 29, 2002.

21. Lee S, Lee HJ, Kim JH, Lee HS, Jang JJ and Kang GH: Aberrant $\mathrm{CpG}$ island hypermethylation along multistep hepatocarcinogenesis. Am J Pathol 163: 1371-1378, 2003. 